\title{
Value-Chain Engineering of a Tower-Top Cellular Base Station System
}

\author{
Tim Cooper and Ronan Farrell \\ Centre for Telecommunications Value-Chain Research (CTVR) \\ Institute of Microelectronics and Wireless Systems \\ National University of Ireland, Maynooth \\ Co. Kildare, Ireland \\ Email: tcooper@eeng.nuim.ie
}

\begin{abstract}
In this paper we present a value chain analysis of mobile 'phone basestations, with a focus on possible antenna based tower-top electronics. Through this analysis we are able to present a number of possible architectural solutions and provide guidance on performance and reliability criterion.
\end{abstract}

\section{INTRODUCTION}

The fundamental layout of the cellular site and base transceiver station (BTS), as shown in figure 1, has not changed significantly in over a decade and is based on mature and stable technology. However, with the advent of each new generation of wireless technology the requirement for hardware compatible with these new standards allows some deviation from this conventional model. Examples of these include; the $3 \mathrm{G}$ Node-B architecture and the base station hotel concept [1]. This allows the BTS may be located many kilometers from the radio frequency (RF) portion of the hardware thereby reducing the cost of deploying a cellular network.

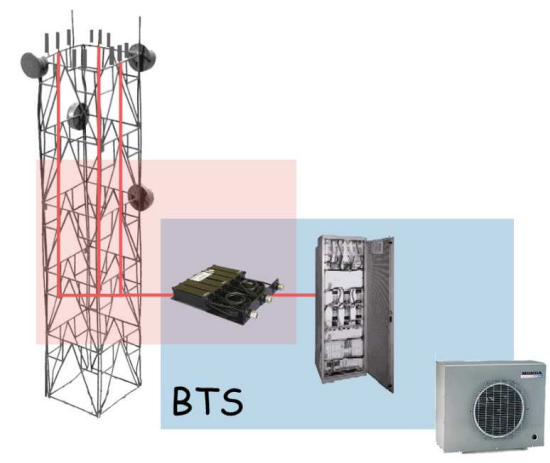

Fig. 1. A representation of the conventional 'tower-bottom' cell site design, the antennas are the only hardware at the tower-top, the rest is located at the tower-bottom in an equipment cabin.

With the objective of further reducing the total cost of ownership of a cellular network, we are investigating the feasibility of tower-top base stations which have the RF portion of the base station electronics relocated to the towertop. Moreover, by considering the impact of this technology on the wireless value chain early on, we hope to add value from the perspective of the BTS manufacturer and network operator by allowing our findings to guide the design and development process.

\section{The Support Services Wireless Value Chain}

The value chain for the provision of wireless infrastructure, at its highest level of abstraction, is shown in figure 2. Here we recognise the distinction drawn by Sabat [2], wherein the production and supply of cellular handsets is an interrelated yet functionally separate value chain, from the support services value chain. Although many BTS manufacturers do also produce mobile handsets, e.g. Nokia, Ericsson and Motorola, it is convenient to limit our analysis to this support services value chain. This chain comprises four broad activities: The first is the supply of information and material through their associated channels to infrastructure providers. Infrastructure provision is the enabling hardware, real estate, back-haul and spectral resources etc. needed to run a communications network. Network provision is the act of supply, maintenance and sale of wireless services, to the source of value, the customer.

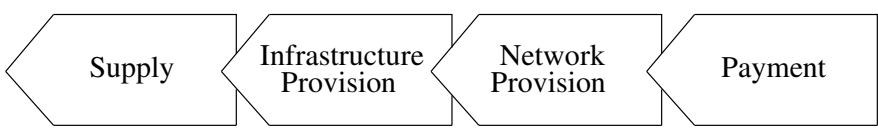

Fig. 2. The support services section of the wireless value chain.

Each link within this support services value chain subsumes a wide variety of activities. Figure 3 shows the activities relevant to this study (for a comprehensive list see [3]). It is clear that the network operator is responsible for the management of many disparate inputs to their value chain. Many of the activities are outsourced and undertaken by single industry firms. For example Alan Dick Co. or Mat Jaybeam in the case of antenna manufacture and American Tower or Crown Castle International in the case of site rental and acquisition. To understand better the impact tower-top relocation may have on the activities within this portion of the wireless value chain it is necessary to introduce our proposed tower-top system.

\section{THE TOWER-TOP BTS}

The conventional layout of a cellular BTS is shown in figure 1. From the transmit perspective, we see there that the 


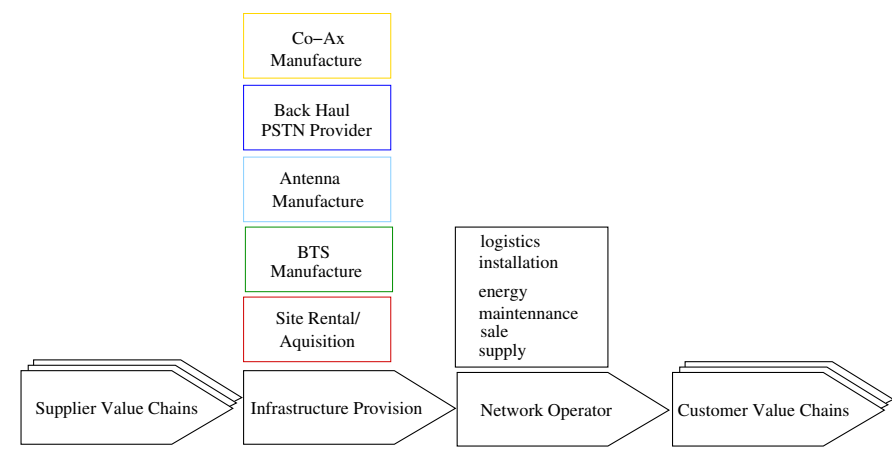

Fig. 3. Examples of relevant activities within the wireless support services value chain.

signal from the base station is sent through a large machined duplexer / combiner system and up co-axial feeder cables to the antennas at the tower-top. Our scheme sees this replaced by the layout shown in figure 4. By distributing the base station electronics at the tower-top the power is combined in the air, allowing the replacement of the machined duplexer with lower cost components. Similarly the RF feeder cables are replaced by a single fibre optic cable. This setup yields many $\mathrm{RF}$ performance advantages and should significantly reduce the cost of hardware production.

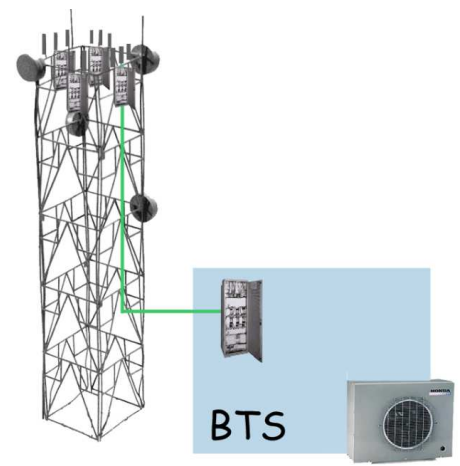

Fig. 4. A representation of a tower-top base-station. Here the electronics are distributed between antenna elements at the tower-top and a single fibre optic cable replaces the co-axial feeder cables.

To illustrate the magnitude of the savings possible consider that typical cavity duplexer filters currently cost $\$ 2250$ each [4], several of which may be employed. If the power radiated by each element were below a certain threshold, these could be replaced by ceramic filters at a cost of $\$ 1.54$ per element [5]. The feeder cables which constitute $8 \%$ of the installation cost of a cell site, and a significant portion of the maintennance cost, may be replaced by a fibre optic cable which, based on a $12 \mathrm{~m}$ monopole mast, could cost $\$ 80$ per site [6], [7].

The principle caveats to these savings are the increase in reliability required of such a system and an enhanced calibration requirement. This calibration requirement already exists to some extent in the specification each network operator or BTS manufacturer passes on to antenna manufacturers, and is typically realised as a passive phasing harness. In a tower-top system this requirement becomes the responsibility of the BTS manufacturer. A detailed technical description of the principle of operation of our patented tower-top calibration scheme is outlined in [8]. The fundamental building block of the system is the 'array element'. This comprises a radio transceiver and antenna element. With a prospective solution to the issue of array calibration, and assuming a standard compliant tower-top transceiver and antenna element can be produced, this leaves the issues of system cost and reliability as our foremost concerns.

\section{Cost And Reliability Study}

\section{A. Assumptions}

To determine the cost and reliability issues facing the towertop base station it is useful to consider them as a function of the number of array elements that are employed. Thus, to limit the investigation to practical, commercially relevant solutions we make the following assumptions and from them determine a rough upper limit on the number of array elements.

- Antenna Size: To remain forward compatible with future smart antenna applications we assume $\lambda / 2$ inter-element spacing. For the purpose of this paper we assume operation in the $2.4 \mathrm{GHz}$ UMTS band. Thus for each element added the array grows by $3.9 \times 10^{-3} \mathrm{~m}^{2}$.

- Cost: If the tower-top system can be built for the same cost as a conventional cellular BTS with equivalent RF and reliability performance, then considerable value will have been added from the viewpoint of the network operator. Based on the analysis conducted in [7] we estimate the cost of a single sector plus attendant radio hardware for a $3 \mathrm{G}$ cell site at $\$ 3,700$. Our system must not exceed this target cost.

- Power: We will restrict the output power of the basestation to $300 \mathrm{~W}$ EIRP. Given that each antenna element will have a gain of $5 \mathrm{dBi}$, this equates to a total of $100 \mathrm{~W}$ at the array feedpoints.

- Tower-loading: Detailed study of tower-loading is not feasible at this early stage. The two primary sources of tower-loading are mass and surface area. As the array must be man portable an upper limit of $30 \mathrm{~kg}$ is imposed. This weight limit means that the system should be installable at the same cost as a conventional antenna. The surface area criterion is based on a Mat-Jaybeam type 5265 cellular sector antenna which has a frontal surface area of $0.77 \mathrm{~m}^{2}$, we set this as our upper array area limit.

Based on the preceding assumptions, we set a provisional upper limit on array size of 100 elements.

\section{B. Results}

Following the imposition of the aforementioned constraints we can draw some initial inferences about our tower-top system. Figure 5 shows the power per element and array area plotted as a function of the number of elements. Note that the 
array area does not exceed our upper limit of $0.77 \mathrm{~m}^{2}$. The three shaded areas on the graph denote differing filter technologies which may be employed at the respective power levels. Starting with the left most, the first indicates cavity based filters. These are large precision engineered units, generally unsuitable for tower-top deployment due to thermal variability and an attendant maintenance requirement. The middle section represents the ceramic based duplexers. These are smaller and may be surface mounted, reducing manufacturing costs and are hence a strong candidate for tower-top use. The third regime is that of relatively low transmit power - here high reliability, very low cost components can be used but requiring many elements.

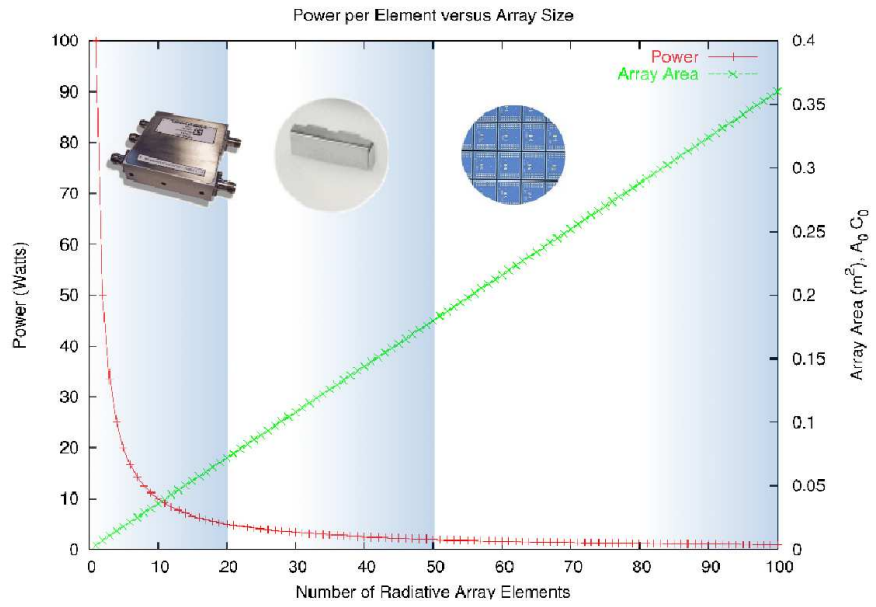

Fig. 5. Technologies suitable for different array sizes, as the array area increases and the power per element drops then lower cost technologies may be availed of.

\section{Costing}

Clearly it would be inefficient and time consuming to generate designs based on each of the three power regimes above. Given that the size available for each element transceiver is roughly the size of a mobile 'phone, figure 6 gives an indication of form factor of a $3 \times 4$ array. Based on these findings, subjective price regimes for each of our three filter technologies were derived. This pricing is based on a combination of mobile 'phone BoM and current transistor power amplifier (PA) pricing.

As we have already stated this estimation is highly subjective and is not a substitute for design, prototype and volume production quotation. Furthermore, the pricing per element is unlikely to be as simple as our piecewise linear approximation shown here. What is apparent, however, is that where there is a boundary between high and low power technology significant advantage may be gained in opting for the lower cost technology and accepting a minor increase in array size. Accepting an increase in array size, especially if it affords redundant elements, may also benefit overall reliability.

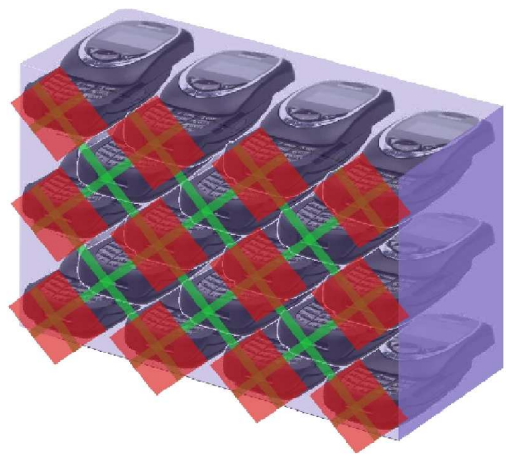

Fig. 6. An indication of the form factor of a twelve element array.

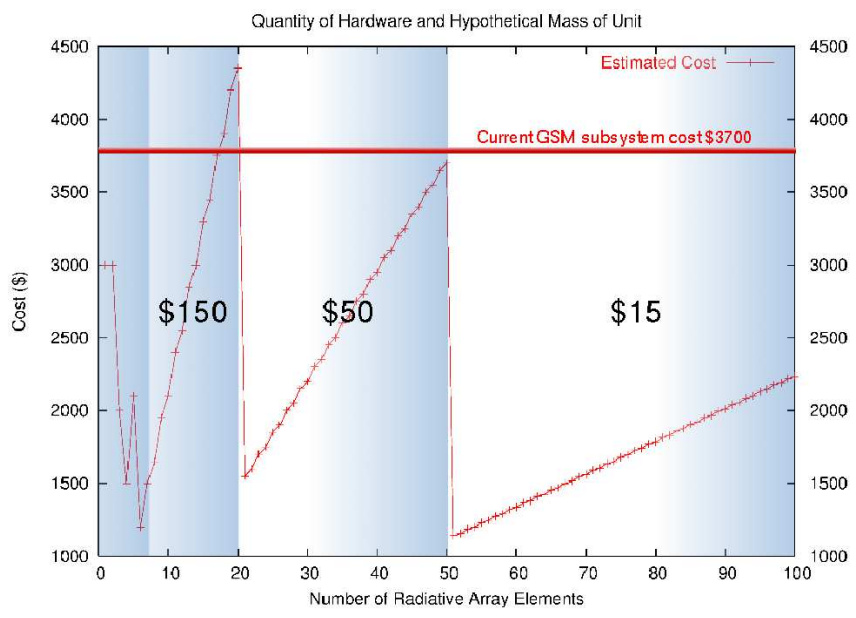

Fig. 7. The effect of price per element on overall system cost. Whilst the cost per element based on our three technologies indicates profitability in all three areas, we focus on the two lower power regimes.

\section{Reliability}

Reliability is a crucial parameter as any failure at the towertop, has not only the direct cost of maintenance, but will also cause dropped calls, customer dissatisfaction and can lead to churn. Although the tower-top unit will be designed to be repairable, by convention we chose mean time to failure (MTTF) as our reliability metric. An example of the requisite reliability demanded of existing tower-top electronics, for example the tower-top LNA, is approximately 28.5 years [9]. In figure 8 we see how an array with extremely high element reliability of 245 years, as the array size increases the time to failure, and hence repair, decays exponentially. It therefore seems hard to conceive of a tower-top system without some level redundancy and each element designed for maximum reliability. It is for this reason that our first line of investigation for a tower-top transceiver will be a direct digital conversion system due to the reduced component count (N.B. there are further cost and reliability ramifications to this choice).

Redundancy will have the effect of increasing reliability, however, the impact of this effect will be less for large arrays. This is offset, of course, by the fact that a lesser fraction 
of the overall array is lost per element failure. Nevertheless, 'soft failure' of the array and redundancy schemes will be necessary in tower-top arrays with a concomitant increase in cost. These factors will remain the subject of future study. The BTS manufacturer must ensure therefore, that to retain value, the service costs will not exceed the savings attainable.

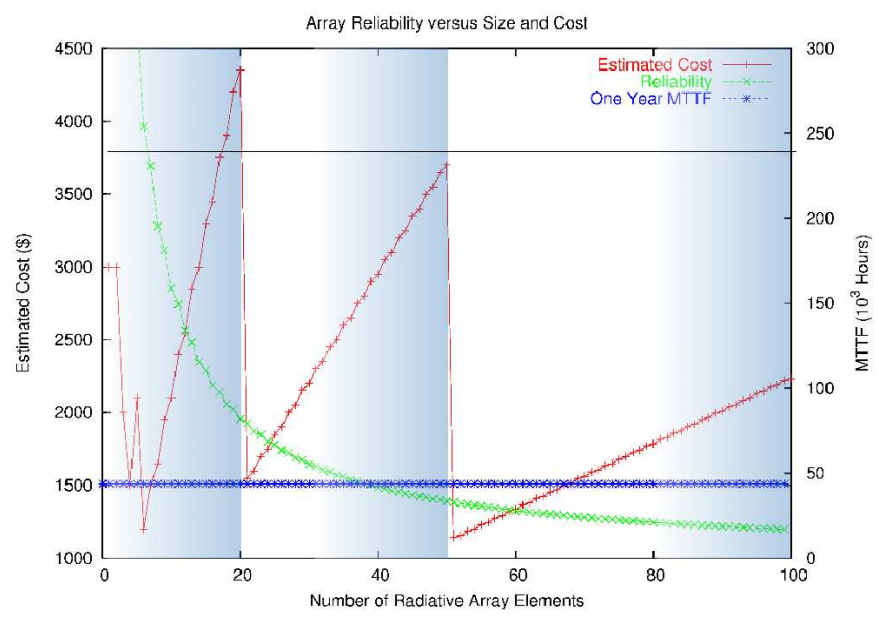

Fig. 8. Reliability shown as a function of array size, superimposed over the previous results.

\section{E. Regimes of Operation}

From the feasibility study of our simplified model, we can see that the existing industry trends, market pressure and available technology give rise to two potential regimes in which the tower-top BTS may prove feasible. The first (20 to 30 elements) is where the power per element is less than $5 \mathrm{~W}$ allowing ceramic filters (e.g. dielectric loaded cavities) to be employed along with high power handset amplifier electronics. The second regime $(50+$ elements $)$ sees less than $2 \mathrm{~W}$ per element power levels and the application of subminiature ceramic filters (e.g. FBAR), extensive CMOS RF circuitry and high reliability / low cost GaAs pHEMT transistor amplifiers. Which regime will yield the optimal reliability, and thus feasibility, remains the subject of continued study.

\section{IMPACT ON THE VALUe Chain}

Assessing the feasibility of tower-top BTS deployment demands more than just maintenance and capital expenditure consideration. Figure 9 shows the value chain of figure 3 modified to reflect the changes bought about by tower-top integration. We emphasise again that this is not every activity within the value chain, but a simplified section - used to highlight some of the key changes in its distribution.

The most prevalent change is the elimination of the co-ax manufacturer. The value from this activity is captured by the BTS manufacturer and the network operator. Other notable changes include the demotion of the antenna manufacturer to a second tier supplier. The antenna module will not require the extensive weather-proof physical housing. This will be added

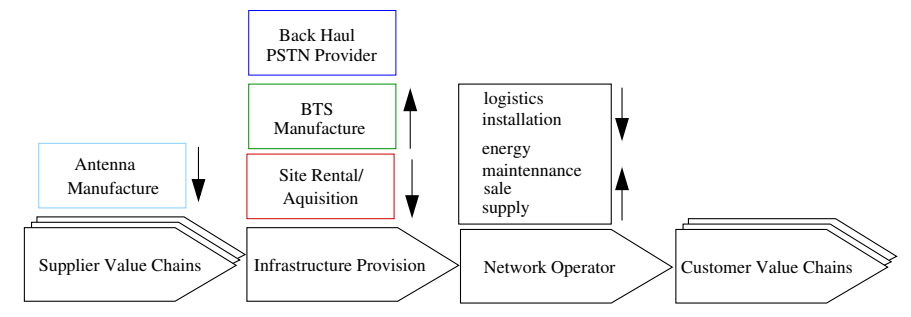

Fig. 9. The modification to activities within the value chain in light of tower-top deployment retaining the conventions of figure 3. Significant value appears to be captured by the BTS manufacturer and reduction in the fixed costs of the network operator.

by the BTS manufacturer - allowing them to capture more value. Some value has been captured from the site owning companies due to limited demand for large ground-level BTS cabinets. Other chains may be largely unaffected, for instance the wireline services may see limited impact from towertop deployment over and above that already seen from the introduction of base station hotels.

Within the activities of the network operator, based on our analysis thus far, the fixed costs of buying and installing a tower-top system will be lower, the only caveat to this is the cost of attaining suitable reliability. If inadequate, this could lead to increased maintenance cost or the addition of more elements for increased redundancy.

There are however other factors worthy of consideration. It is feasible that a tower-top array may be cheaper to run in the face of increasing energy costs. Current BTS efficiency is approximately $2 \%$, i.e. for every watt of AC power $20 \mathrm{~mW}$ of RF are generated [10]. Considerable efficiencies should be achieveable as the three least efficient components, the HVAC, PA and feeder cable are all either eliminated or higher efficeiency. From the feeder alone savings of $50 \%$ per site per anum will be attained.

\section{TOtAl COST OF OWNERShiP}

To help present these different issues in context, the following table compares the total cost of buying and operating our tower-top scheme (in each of the three regimes identified over a ten year life span) with that of a conventional towerbottom system. The first section of the table compares the capital cost of the hardware. The tower-bottom base station figures are derived from those in [4], [7], [10]. The cost of the tower-top hardware is based upon the three worst predicted prices from our three power regimes. As a precaution we have also added $\$ 1500$ for additional tower-bottom hardware. Furthermore, the duplexer price is added to the total cost. In spite of this conservative pricing assessment, substantial capital savings are possible (shown in the centre of the table).

The lower section of the table shows the operational expenses of energy and maintenance for the four schemes. Due to the lack of reliability information for the tower-top system the service cost is treated as unknown. However, from the total capital savings, and assuming an annual maintenance budget 


\begin{tabular}{|c|c|c|c|c|}
\hline & Tower-Bottom & \multicolumn{3}{|c|}{ Tower-Top } \\
\cline { 3 - 5 } Item & & 20 element & 50 element & 100 element \\
\hline Tower-Top Unit & 1200 & 13200 & 11100 & 6600 \\
Tower-Bottom Unit & 15000 & 1500 & 1500 & 1500 \\
Cabling & 20000 & 80 & 80 & 80 \\
Duplexor & 7000 & 900 & 680 & 460 \\
\hline \hline Total & 43200 & 14870 & 13360 & 8640 \\
\hline Saving (Capex) & 0 & 28330 & 29840 & 34560 \\
\hline \hline Energy & 195000 & 97500 & 97500 & 97500 \\
Service & 100000 & $?$ & $?$ & $?$ \\
\hline Service Budget & 100000 & 225830 & 227340 & 232060 \\
\hline
\end{tabular}

\section{ACKNOWLEDGMENT}

The authors would like to thank the generous funding of this project by Science Foundation Ireland through the Centre for Telecommunications Value Chain Research.

\section{REFERENCES}

[1] A.J. Cooper, Fibre-radio for the provision of cordless/mobile telephony services in the access network, Electron. Lett. vol 26 p.p. 2054-2056, 1990.

[2] H.K. Sabat, The evolving telecom value chain and market structure, Telecom. Policy (Special Issue) vol 26 No. 9-10, p.p. 505-535, 2002.

of $\$ 10000$ per site for the tower-bottom system [11], service budgets which allow the tower-top scheme to break-even may be predicted. These are over twice that of a conventional site and are shown on bottom row of the table. Thus providing both a rationale for the change in architecture and a reliability design target for system developers.

\section{CONCLUSION}

From this analysis we conclude that a ten year service budget of approximately $\$ 220000$ per site allows tower-top technology to break even with current technology. Should comparable service cost to that currently achieved in a towerbottom system be attained, substantial savings for the network operator are possible. This insight into the profitable regimes of tower-top operation and the importance of reliability will allow us to guide the development of the tower-top system and maximise the value it adds to the wireless value chain.

[3] S.C. Constance, J.R. Gower, A Value Chain Perspective on the Economic Drivers of Competition in the Wireless Telecommunications Industry, Masters Thesis, Massachusetts Institute of Technology, June 2001.

[4] Wainwright Instruments Gmbh., Unit price of: WDG 880/915-925/96075/20, Andechs-Frieding, Germany.

[5] Avago Technologies, Unit price of: FBAR ACMD-7401, San Jos, CA, USA.

[6] Control Cable Inc., Pricing: Mohawk M9B511T, Baltimore, MD, USA.

[7] Unstrung Insider Report, Open basestation bonanza, vol 4 p.p. 1-20, May 2005.

[8] T.S. Cooper, G. Baldwin and R. Farrell, Toward scalable, automated tower-top phased array calibration, (submitted) VTC 2007.

[9] ADC SMATop Tower-Top Amplifier, Target MTTF of:DD1900, ADCP75-103, Issue 3, p. 27, March 2001.

[10] T. Elder, S. Lundberg, Energy efficiency enhancements in radio access networks, Ericsson Review, No. 1, p.p. 42-51, 2004.

[11] Vodafone Vodafone Annual Report, Newbury, Berks., UK, 2005. 